\title{
The Search for pulsed TeV gamma-ray sources
}

\author{
Andrew McCann ${ }^{1} \dagger$, Michael Daniel ${ }^{1}$, David J. Fegan ${ }^{1}$, \\ Stephen Gammell, Peter Cogan ${ }^{1}$ and John Quinn ${ }^{1}$
}

${ }^{1}$ School of Physics, University College Dublin email: andrew@ferdia.ucd.ie

\begin{abstract}
The EGRET experiment detected pulsed emission from several pulsars in the HE energy range $20 \mathrm{MeV}<\mathrm{E}<10 \mathrm{GeV}$. However, no pulsed emission has ever been detected above $300 \mathrm{GeV}$, which points to a cut-off in pulsed emission somewhere between $\sim 10 \mathrm{GeV}$ and $\sim 300 \mathrm{GeV}$. Software has been developed at UCD which is capable of examining gamma-ray events in the energy range $E>130 \mathrm{GeV}$ and detecting the signature periodicity of pulsars if it exists. Data recorded on the Crab pulsar with the Whipple Imaging Atmospheric Cerenkov Telescope has been analysed with this software and upper limits on pulsed emission have been obtained.
\end{abstract}

\section{Introduction}

The low flux of gamma rays above $10 \mathrm{GeV}$ and the small collection area of gamma-ray satellites means that there have been no detections of pulsars above $10 \mathrm{GeV}$. The Imaging Atmospheric Cerenkov Technique (IACT) has limited sensitivity at energies below $\sim 300 \mathrm{GeV}$ due to effects of the cosmic-ray background. However, it has a proved successful at detecting $\mathrm{TeV}$ gamma rays. To this date there has been no detection of $\mathrm{TeV}$ gamma-ray emission from pulsars by the IACT, which suggests that the emission mechanisms responsible for pulsed gamma rays are halted between $\sim 10 \mathrm{GeV}$ and $\sim 300 \mathrm{GeV}$. This cut-off is a prediction of all current pulsar emission models, however the energy at which the cut-off occurs is unique to each model. Experimental determination of the cut-off energy, and its correlation with a particular theoretical model, provides the motivation for this work. For a review of pulsar emission models see Baring (2004) and Hirotani (2005) .

In this work data on the Crab pulsar, taken with the Whipple Telescope (Finley et al., 2000), has been analysed with a gamma-ray selection procedure called Kernel analysis (Dunlea et al., 2001). This procedure has proved successful at detecting gamma rays close to the telescope's energy threshold.

\section{Results}

No pulse profile is evident in the lightcurves determined from the gamma-ray data (see Figure 1) and $\chi^{2}$ tests yielded no deviation from a uniform distribution. Using the method of Helene (1983), upper limits on peak area were determined, with the assumption that phases of emission at $\mathrm{TeV}$ energies are the same as those detected by EGRET. These upper limits were subsequently converted to flux upper limits, yielding an integral flux upper limit of $5.8 \times 10^{-7} \mathrm{~m}^{-2} \mathrm{~s}^{-1}$ for $\mathrm{E}>130 \pm 39 \mathrm{GeV}$. This upper limit is compared with EGRET data and previous IACT investigations in Figure 2.

$\dagger$ Present address: School of Physics, University College Dublin. 

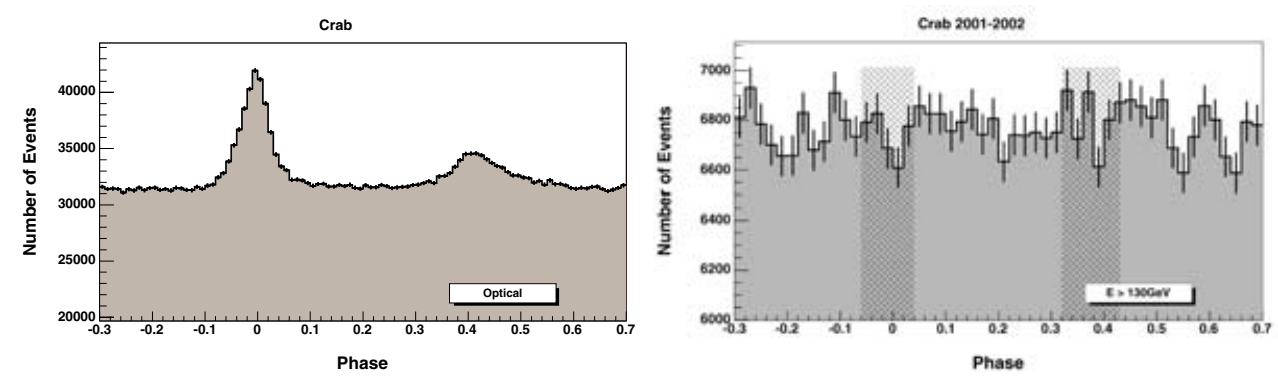

Figure 1. Left: The optical lightcurve of the Crab pulsar determined from data taken with the Multiple Mirror Telescope Right: Crab pulsar data taken with the Whipple telescope between 2000-2001 (E > $300 \mathrm{GeV})$. Both datasets were analysed by the same method. No pulse is evident in the gamma-ray data.

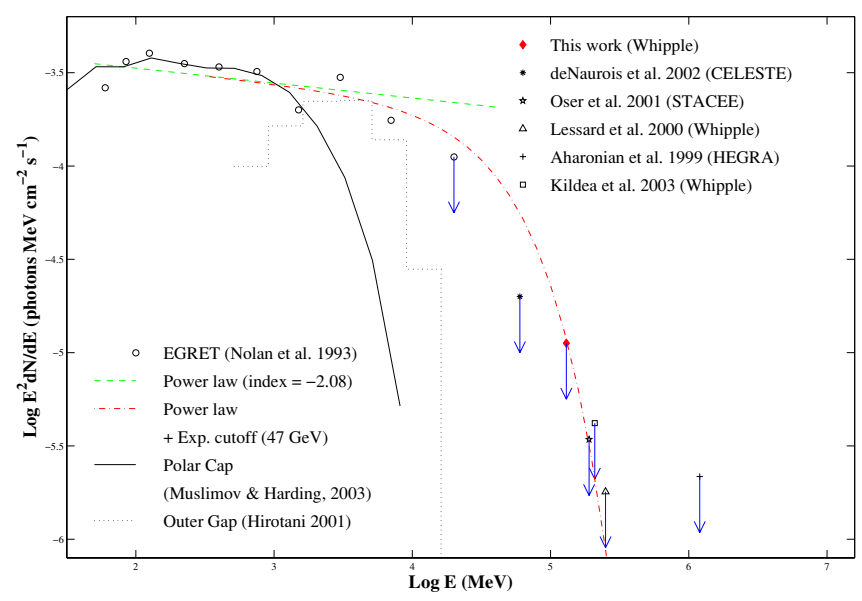

Figure 2. Pulsed photon spectrum of the Crab pulsar. Originally from Kildea et al. (2003).

\section{Conclusion}

This work has successfully probed pulsar emission at energies closer to the Whipple telescope energy threshold than any other investigation. This was made possible by applying a low-energy Kernel selection developed at UCD. In spite of these advances the results presented do not constrain any mainstream models of pulsed emission and it is clear that a further reduction in energy threshold will be required to do so. Other candidates for pulsed TeV emission are currently under study.

\section{References}

Baring, M.G. 2004, Advances in Space Research, 33, 552

Dunlea, S. et al., 2000, in ICRC, Vol. 7, p. 2939

Finley, et al., 2000, in American Institute of Physics Conference Series, p. 301

Kildea, J., Gammell, S., and VERITAS Collaboration 2003, in ICRC, p. 2377

Helene, O. 1983, Nuclear Instruments and Methods 212, 319

Hirotani, K. 2005, Astrophysics and Space Science, 297, 81 